\title{
MALARIA AMONG FOREIGN WORKERS IN SELANGOR, MALAYSIA
}

\author{
Masitah $M^{1}$, Nor Aini M N ${ }^{2}$, Mas Ayu $\mathrm{S}^{3}$ \\ 1 AIDS/STI Section, Ministry of Health, Malaysia \\ 2 Vector Borne Disease Control Unit, Selangor State Health Department, Selangor. \\ 3 Department of Social Preventive Medicine, Faculty of Medicine, University of Malaya, Kuala Lumpur.
}

\begin{abstract}
:
The state of Selangor, Malaysia, is facing a significant rise in the number of malaria cases with the incidence varying from 20 to 90 per 100,000 persons in a population. A study was carried out to describe the distribution of malaria cases in relation to the growing number of foreign workers in Selangor. Data were reviewed using the return forms "PBV (M) 101", a summary of malaria cases in Selangor for 2006 and Annual Reports of Vector-Borne Disease Control Unit, Selangor State Department for 2001-2005. The malaria cases in Selangor varied between 172 cases in 2001 and 90 cases in 2006. Most of the cases were contributed by foreign workers, who were predominantly male of economically active age group and came from malaria endemic countries. Most of these cases were concentrated in the urban districts. Malaria is still endemic in Selangor. Malarial infection has the potential of contributing to an urban outbreak of malaria. (JUMMEC 2008; 11 (2):53-58)
\end{abstract}

KEYWORDS: malaria, incidence, foreign workers, imported, outbreak

\section{Introduction}

Malaria is one of the re-emerging diseases in Selangor, Malaysia, and the incidence is increasing due to of several reasons such as anti-malarial drug resistance, insecticide resistance and international travel $(1,2)$. Currently, malaria is still a public health problem in Malaysia $(2,3,4,5)$. Since the implementation of the Malaria Eradication Program in 1967 that was later converted to the Vector-Borne Disease Control Program in the 1980's, the number of malaria cases has declined significantly. Within the past 10 years, the incidence rate has declined significantly from 300/100,000 population in 1994 to $24.1 / 100,000$ population in the year $2004(3,4)$ (Figure 1).

Malaria cases were commonly reported in the rural areas (86.5\%) and among the Orang Asli (33.1\%) (4). However, new sources of contracting malaria have been introduced recently. The sources of infection in these areas were noted to be from foreign workers ( 2 , $5,6)$. Malaysia is highly dependent on foreign unskilled and semiskilled labourers. There were 1.7 million legal migrant workers in Malaysia in 2005 (7). Of concern, most of these foreigners came from malaria endemic countries $(2,3)$.
WHO reported that there were over 2 million cases of malaria in India, over 200,000 cases in Vietnam and over 100,000 cases in Myanmar and Pakistan in 1992 (2). The Ministry of Health of Indonesia reported that there were over 3 million clinical malaria cases in 1998 (8). These migrants may carry with them microbial pathogens. In addition, their cultural traditions and behavioural patterns promote disease transmission, as does living in unhealthy and crowded slum conditions $(2,6)$.

The incidence of malarial infection among foreign workers has been steadily increasing. Several focal outbreaks were reported among foreign workers who worked in the construction sites in the urban areas $(4,6)$. The total number of cases among immigrants has increased from 1,627 cases in 2003 to 2,009 cases in 2004 . Some $74.2 \%$ of these cases were from

\section{Correspondence:}

Masitah Mohamed

Department of Social and Preventive Medicine

Faculty of Medicine, University of Malaya

50603 Kuala Lumpur

Email: itaimran@yahoo.com.my 


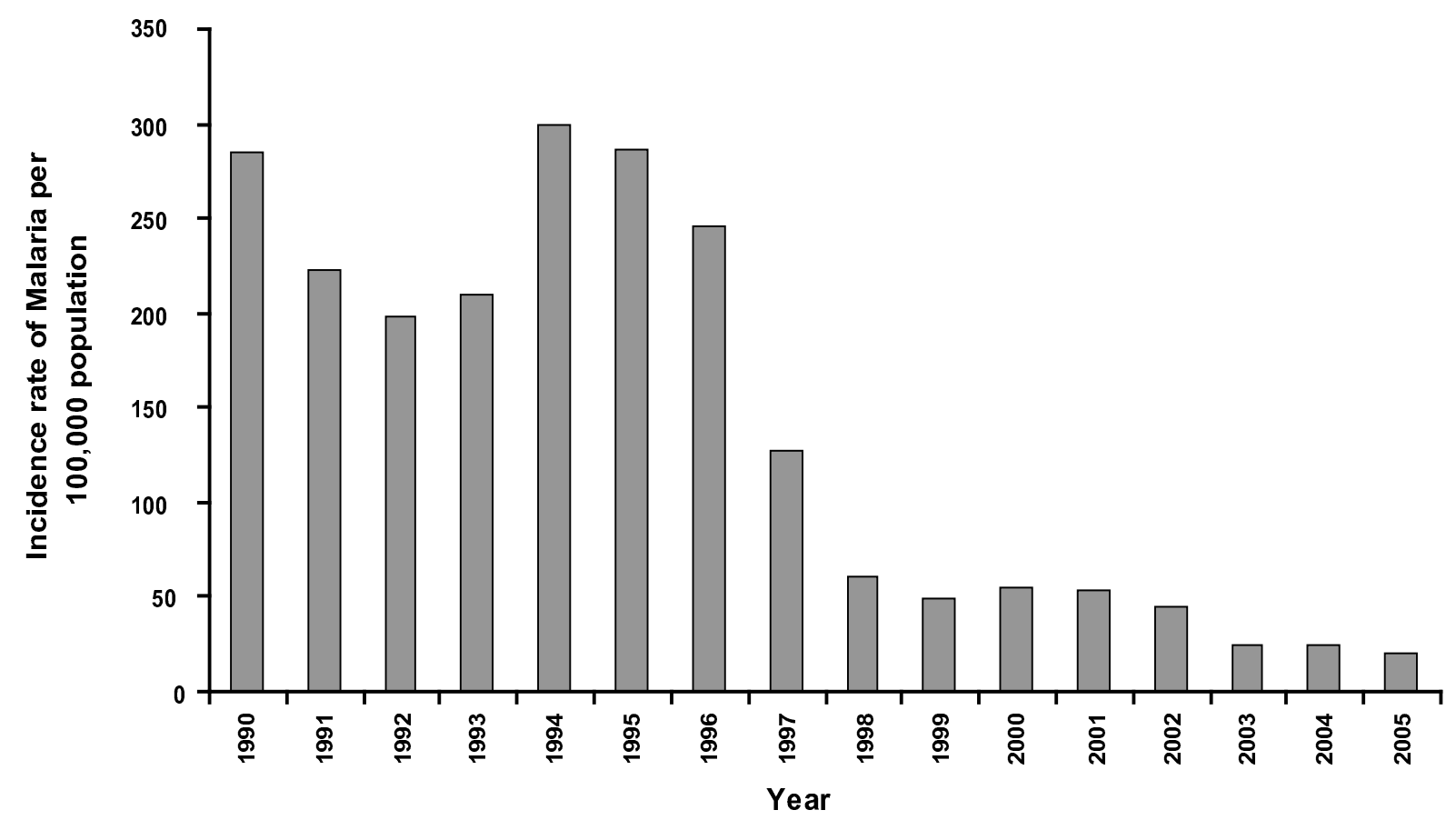

Figure 1: Incidence of Malaria, Malaysia, 1990-2005

Indonesian workers. These cases contributed to about $32.6 \%$ of the total reported cases of malaria in the year 2004 (4).

Malaria is a vector borne disease transmitted to man by the bite of an infected anopheline mosquito. The causative agent of malaria is a protozoan of the genus Plasmodium, and four species affect humans, i.e. $P$. falciparum, $P$. vivax, $P$. ovale and $P$. malariae (9, $10,11)$.

If severe cases of malaria were not managed properly they would lead to complications, such as cerebral malaria or death $(11,12)$. The case fatality rate for malaria in Malaysia has increased from $0.33 \%$ in 2003 to $0.58 \%$ in 2004 (4). Therefore, there is a need for information on the characteristics of malaria cases to help in the control of this disease.

Selangor is one of the most developed and rapidly urbanising states in Malaysia. It covers 7960.84 km² $^{2}$ area with a population of about 4.7 million in the year 2005 (13). However, Selangor still faced a significant number of malaria cases with the incidence rate of malaria fluctuating from 20 to 90 per 100,000 populations between 1996 and 2006. Of concern, these cases were commonly reported in urban districts such as Petaling, Hulu Langat and Gombak, which have high population densities (Figure 2)
This study described the epidemiology of malaria in Selangor with special reference to foreign workers.

\section{Methods}

This was a descriptive study mainly to describe the trend of malaria cases for the past six years. The characteristics of malaria cases in 2006 were obtained from reviewing secondary data, such as the malaria returns forms "PBV (M) 101," summary of malaria cases in Selangor for the year 2006 and Annual Reports of Vector-Borne Disease Control Unit (2001-2005) from the Selangor state health department.

The PBV (M) 101 or Malaria Case Registry form contains information about the malaria cases from a particular district or state. Information reported in this form include socio-demographic characteristics of the patients, investigation and treatment aspects of each malaria case.

The case definition for malaria in this study was "any foreign worker with or without symptoms or signs of malaria who shows laboratory confirmation of parasitaemia" (14). Based on the criteria set by the Ministry of Health, "Imported A" was defined as "any malaria infection where the source of the infection was imported from outside this country". The patient must have contracted the infection within 


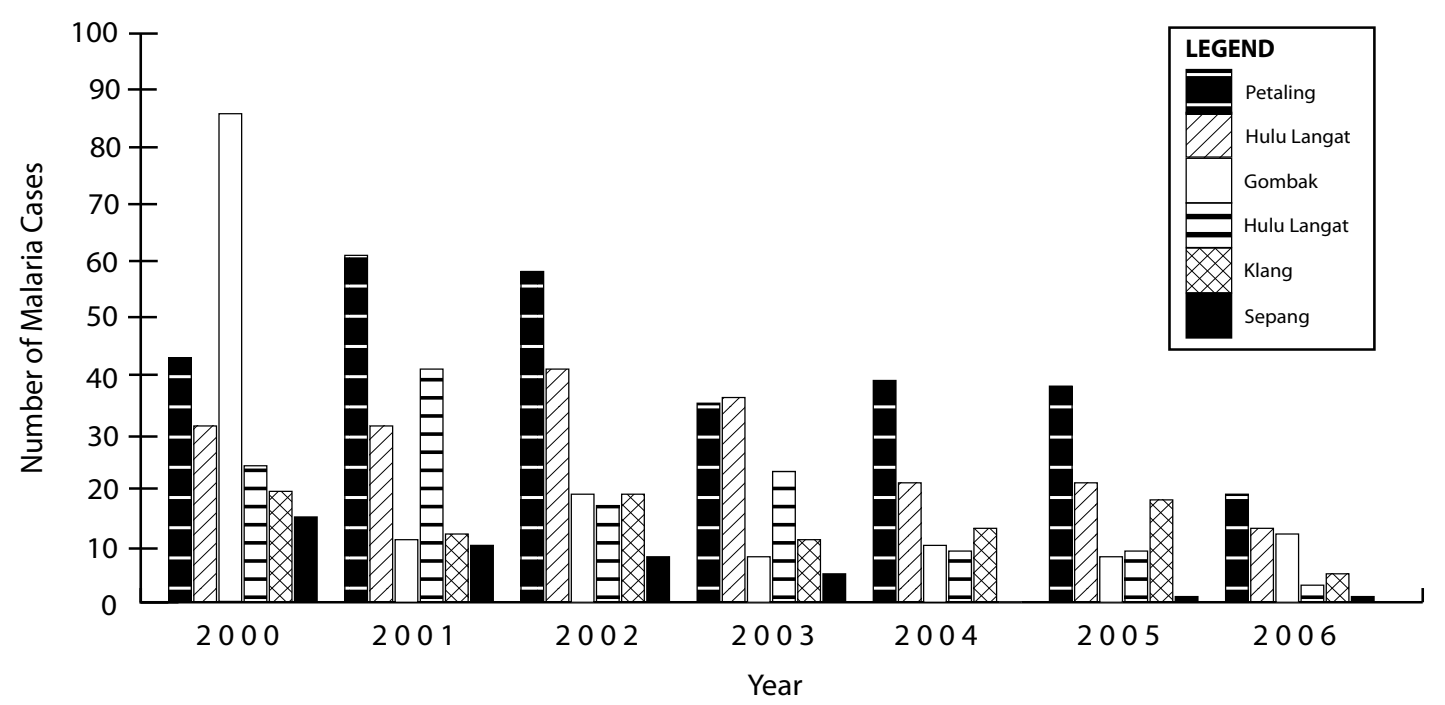

Figure 2: Distribution of Malaria Cases by Selected Districts, Selangor, 2000-2006

three months of staying in Malaysia and his/her place of stay in Malaysia is a malaria risk area.

The variables included for this study were age, gender, citizenship status, type of infection, source of infection and reporting district. Data were analysed using the statistical package for social science (SPSS) 12.0 for Windows (15). After the data entry, data cleaning was conducted. This involved checking the frequency of all the variables. All data were shown as numbers and proportions.

\section{Results}

Malaria cases were still endemic in Selangor. The number of malaria cases in Selangor varied between 172 cases in 2001 and 90 cases in 2006. On further analysing the distribution of these cases, it was evident that there was a significant contribution from foreign workers to the total number of cases. The proportions of foreign workers have increased from $57 \%$ to nearly $75 \%$ out of the total number of malaria cases in Selangor in the years 2001-2006 (Figure 3).

The workers were predominantly male ( $92.7 \%)$. The median age was 26 years old (interquartile range: $23-$ 31) (Figure 4).

The distribution of these cases according to countries of origin showed that most of the cases were from Indonesia (17 cases) and Myanmar (16 cases), followed by India ( 8 cases) and Pakistan (7 cases) (Figure 5).

The source of infection (89.1\%) was imported from other countries based on the criteria set by the Ministry of

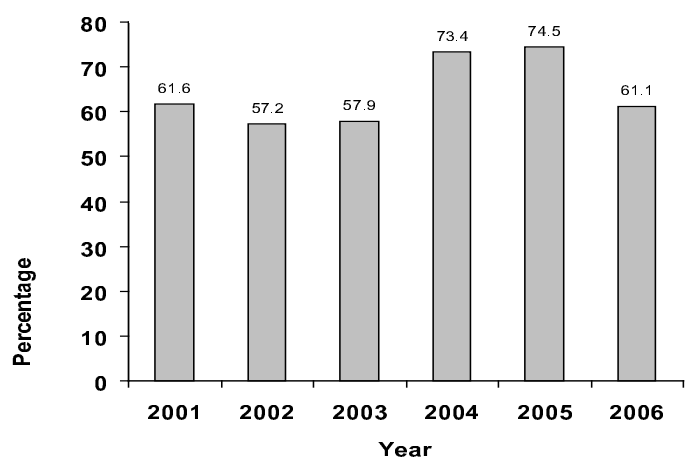

Figure 3: Proportion of Malaria Cases Contributed by Foreign Workers, Selangor, 2001-2006

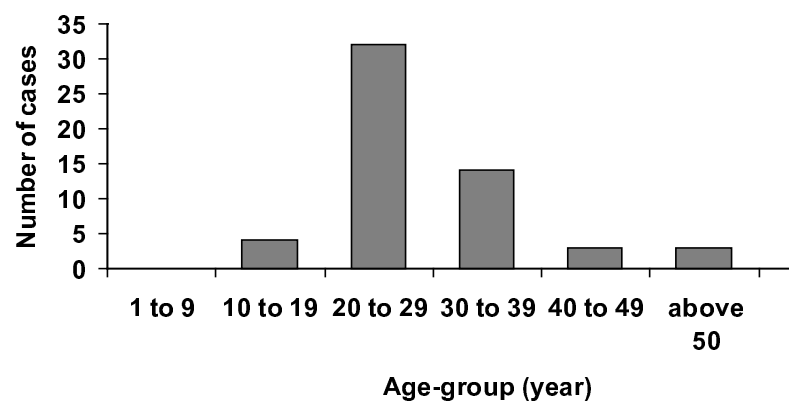

Figure 4: Distribution of Malaria Cases (Foreign Workers) by Age Groups, Selangor, 2006

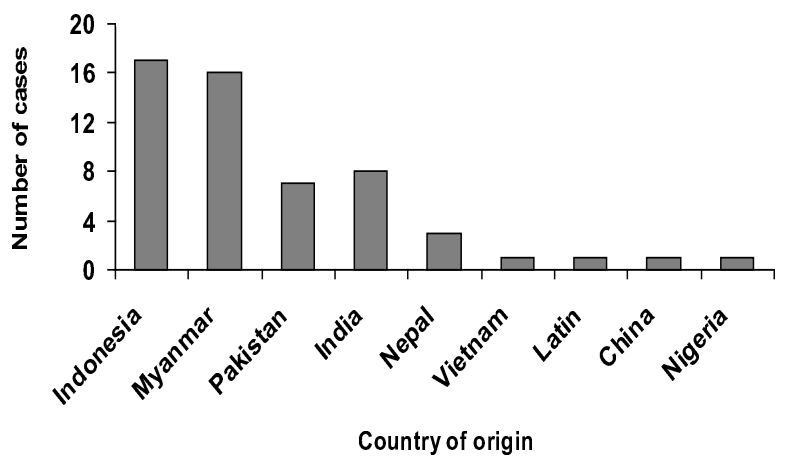

Figure 5: Distribution of Malaria Cases (Foreign Workers) by Countries of Origin, Selangor, 2006 
Health (imported A). Most of them were infected by Plasmodium vivax (70.9\%), followed by Plasmodium falciparum (27.3\%) and Plasmodium malariae (1.8\%). Distribution of the cases according to districts in the state of Selangor, Malaysia showed that most of them were from Petaling (36.5\%), Hulu Langat (23.1\%), Gombak (23.1\%), Klang (9.6\%), Hulu Selangor (5.8\%) and Sepang (1.9\%).

\section{Discussion}

Overall, this study showed that malaria cases still exist in Selangor despite the fact that it is one of the most urbanised states in Malaysia. About $60 \%$ of the malaria cases reported in Selangor were significantly contributed by foreign workers who were predominantly males of economically active age group. Many of these workers came to Malaysia to work as unskilled and semi-skilled labourers.

An important point to note is that these cases came from malaria endemic countries such as Indonesia, India, Vietnam, Myanmar and Pakistan $(2,16)$. Since these workers are young, active, and highly mobile, they have the potential to transmit infections to others.

The proportion of cases actually corresponded with the distribution of migrant workers in Malaysia: Indonesian workers were the highest (68.9\%), followed by others such as Nepalese (9.9\%), Indian (6.9\%) and Myanmar (4.6\%) (16). Previous studies have also shown that a significant proportion of migrant workers in referral hospitals $(5,17)$. A retrospective survey in the University Hospital Kuala Lumpur in 1998 showed that out of 134 malaria patients who were admitted throughout the period 1983-1992, foreigners made up $32.8 \%$ of all the malaria cases. Indonesian workers contributed $16.4 \%$ of all these cases (5).

Another survey, which was carried out in the University Hospital Kuala Lumpur showed that out of 81 cases of malaria which were admitted to that hospital, $49 \%$ of them were imported malaria (17).

The Foreign Workers Medical Examination Monitoring Agency (FOMEMA) stipulated a set of laboratory examinations that are mandatory to be carried out on foreign workers. The laboratory examinations include tests for HIV, hepatitis B, malarial parasites and others.
Those who failed the medical tests were deported and their work permits were revoked (7). However, these examinations may not have detected the incubating or dormant states of malarial infection because the examination was conducted by testing blood film for malarial parasite (BFMP) $(2,18,19)$.

In addition, since the commonest infection detected in these foreigners was Plasmodium vivax that has the tendency to relapse, these cases might not have been detected during the screening examination. In this type of infection, relapse can occur months or years after exposure due to the presence of latent parasites in the liver $(10,20)$.

On top of that, there was also no examination conducted on illegal workers, who were believed to be present in a significant number, who might be having this infection and transmitting this disease to others (7).

This finding is consistent with the distribution of malaria cases in Malaysia, which showed Plasmodium vivax as causing the highest infection, i.e. $51.6 \%$ of the cases. On top of that, Asia is one of the regions that is contributing to the largest number of vivax malaria cases in the world (20).

One of the new techniques that is available to detect malarial infection in the latent or recrudescence stage of disease is polymerase chain reaction (PCR), which is capable of detecting parasites at a density of 1 per microlitre or less compared to a microscope, whose threshold of detection is around 10-50 parasites per microlitre. It is an expensive test. However, in the long run it may be more cost effective in detecting malaria cases.

Of concern, these malaria cases were commonly reported in the urban districts of Selangor that have a higher density of population, such as the Petaling, Gombak and Hulu Langat districts. This was because these districts have a lot of work opportunities for the migrant workers either in the factories, housing area or plantation sites. Therefore, the potential threat of urban outbreak of malaria cannot be ignored.

\section{Study Limitations}

There were limitations of this study that need to be considered while interpreting the results. Firstly, the 
study was a review of surveillance data. The captured information was aggregated and not the individual characteristics of the cases.

In this study, the incomplete information included occupation and entry status of the foreign worker (legal or illegal). The entry status is crucial to ensure that medical examination has been conducted prior to entry. The observation seen in this study might be only the tip of the iceberg and the actual number of cases might be more because illegal migrants never turned up for treatment.

\section{Recommendation}

Decisions and actions to control the potential spread of malarial infection by migrants must be based on properly collected data. The malaria returns form should include further detailed history such as district of origin, duration of stay in Malaysia and status of the migrant (legal or illegal). The past medical history should include history of malarial infection, history of contact to malaria cases, drug history and history of examination by FOMEMA.

It is important to have earlier detection and notification of these cases so that epidemiological investigations can be done to ascertain the source. This study only looked at the descriptive epidemiology of malaria in Selangor. Further study is needed to look into risk factors for malaria transmission among these foreign workers and comparing them with factors for local population.

\section{Conclusion}

Malaria is still endemic in the state of Selangor, Malaysia. This is actually due to imported malarial infection. Infections among the foreign workers have the potential of spreading the malaria diseases to urban areas and in contributing to an outbreak. Knowing the risk factors that lead to transmission of infection in these migrants could contribute significantly to the prevention of malaria in this country.

\section{References}

1. Krogstad DJ. Malaria as a re-emerging disease. Epidemiol Rev 1996;18:77-89.

2. Lim VKE. Imported infections. (Editorial). Med J Malaysia 1998;53 (1):1-3.
3. Ministry of Health. Malaysia's Health 2002. Technical report of the Director-General of Health Malaysia. Ministry of Health Malaysia, 2002.

4. Ministry of Health. Annual report 2004. VectorBorne Disease Section. Ministry of Health Malaysia; 2004.

5. Jamaiah I, Anuar AK, Najib NARN, Zurainee MN. Imported malaria: a retrospective study in University Hospital Kuala Lumpur, a ten-year experience. Med J Malaysia 1998;53: 6-9.

6. Wong $\mathrm{YL}$, Mohd Amin J. Malaysian Society and health issues and challenge in the 21 st Century. JUMMEC 2003-2005;8:2-8.

7. Jayakumar G. Pre-employment medical examination of migrant workers-The ethical and iegal issues. Med J Malaysia 2006; 61(5):516-518.

8. Syafruddin D, Asih PBS, Siregar J E Molecular basis of antimalarial drug resistance in Indonesia. In: Tropical Diseases. From Molecule to Bedside. New York: Kluwer Academic/Plenum Publishers, 2003; 103-112.

9. Ministry of Health. Guidelines for the management of malaria. Vector Borne Diseases Control Programme, Ministry of Health Malaysia; 1-40.

10. Fernando RL, Fernando SSE, Leong ASY. Tropical Infectious Diseases: Epidemiology, Investigation, Diagnosis and Management. London: Alden Press Ltd., 2001; 130-200.

11. Griffith KS, Lewis LS, Mali S, Parise ME. Treatment of Malaria in the United States: A Systemic Review. JAMA 2007; 297:2264-2276.

12. Sachdev HS, Mohan M. Vivax Cerebral Malaria. J Trop Paediatr 1985; 31:213-215.

13. Selangor State Department. Annual Reports of Vector-Borne Disease Control Programme. 20012005.

14. Ministry of Health. Case definitions for infectious diseases in Malaysia. Surveillance for infectious disease. Disease Control Division. 2nd ed. Ministry of Health Malaysia; Jan.2006.

15. Sheridan JC, Lyndall GS. SPSS. Analysis without anguish. Version 11.0 for Windows. Australia: Wiley John Wiley \& Sons Australia Ltd., 1999; 1-57. 
16. Vijayakumari K. Towards an East Asian cooperation framework for migrant abour. Migrant workers in Malaysia: An Overview. Internet communication. http://www.isis.org.my/files/pubs/papers/VK_ MIGRATION-NEAT_6Dec06.pdf. 2 Mac 2007.

17. Moore CS, Cheong I. Audit of imported and domestic malaria cases at Kuala Lumpur Hospital. Br Clin Pract 1995; 49(6):304-307.
18. Greenwood B. the Molecular Epidemiology of malaria. Trop Med Int Health 2002; 7(12):1012-1021.

19. Muhlberger N, Jelinek T, Gascon J. Epidemiology and clinical features of vivax malaria imported to Europe: Sentinel surveillance data from TropNetEurop. Malaria J 2004; 3(5):1-7.

20. Lim HC, Mary EW, Patricia S. Prevention in longterm travellers. JAMA 2006; 296(18):2234-2244. 